\title{
COPPER NANOPARTICLE CATALYZED REGIOSELECTIVE CYCLOADDITION OF TERMINAL ACETYLENES TO SODIUM AZIDE IN THE PRESENCE OF $O$-TOSYL FLUOROCARBOHYDRATE DERIVATIVES: ASSEMBLY OF NOVEL ENANTIOPURE FLUOROMONOSACCHARIDES WITH 1,2,3-TRIAZOLE AND 4-PHENYL-OXAZOLIDIN-2-ONE SUBUNITS
}

\author{
Ahlem Guesmi ${ }^{1,2}$, Naoufel Ben Hamadi ${ }^{2,3, *}$ \\ ${ }^{1}$ Textile Engineering Laboratory, Higher Institute of Technological Studies of Ksar Hellal, \\ University of Monastir, Tunisia \\ ${ }^{2}$ Al Imam Mohammad Ibn Saud Islamic University (IMSIU), College of Sciences, Department of Chemistry, \\ 11623 Riyadh, Saudi Arabia \\ ${ }^{3}$ Laboratory of Heterocyclic and Natural Substances Synthesis, Department of Chemistry, \\ Faculty of Sciences of Monastir, University of Monastir, Boulevard of Environment, 5000 Monastir, Tunisia \\ "bh_naoufel@yahoo.fr \\ A three-component reaction of $O$-tosyl carbohydrate derivatives with sodium azide and terminal \\ alkynes can be catalyzed by copper nanoparticles under ambient conditions. A series of 1,4-disubstituted- \\ 1,2,3-triazoles were obtained regioselectively by this one-pot strategy in good to excellent yields \\ (70-90\%). The salient features of the present protocol are mild reaction conditions, a shorter reaction \\ time, and its applicability to a wide range of substrates.
}

Keywords: carbohydrates; terminal alkyne; copper nanoparticles; cycloaddition; 1,2,3-triazoles

\section{РЕГИОСЕЛЕКТИВНА ЦИКЛОАДИЦИЈА НА ТЕРМИНАЛНИ АЦЕТИЛЕНИ СО НАТРИУМ АЗИД ВО ПРИСУСТВО НА $О$-ТОЗИЛ-ФЛУОРОКАРБОНАТНИ ДЕРИВАТИ КАТАЛИЗИРАНИ СО БАКАРНИ НАНОЧЕСТИЧКИ: \\ СОСТАВУВАњЕ НА НОВИ ЕНАНТИОЧИСТИ ФЛУОРОМОНОСАХАРИДИ СО 1,2,3,-ТРИАЗОЛНИ И 4-ФЕНИЛ-ОКСАЗОЛИДИН-2-ОНСКИ ПОДЕДИНИЦИ}

\begin{abstract}
Трокомпонентната реакција на $O$-тозилокарбонатни деривати со натриум азид и терминални алкини може да се катализира со бакарни наночестички во амбиентални услови. Со оваа стратегија е добиена региоселективна серија во еден реакциски сад со добар до одличен принос (70-90\%). Главните карактеристики на овој протокол се благите реакциски услови, покусото време на реакција и применливоста на широк опсег на супстрати.
\end{abstract}

Клучни зборови: јаглеводороди; терминални алкини; бакарни наночестички; циклоадиции; 1,2,3-триазоли

\section{INTRODUCTION}

Substrates containing an acetylene moiety are valuable building blocks for the synthesis of triazole derivatives [1] using 'click chemistry'.
Many of the triazole derivatives find extensive applications in the field of materials science [2]. Complex molecules containing a triazole unit act as glycosidase inhibitors, HIV protease inhibitors, cell imaging agents and G-protein coupled receptor 
ligands [3]. Therefore, the synthesis of various triazole derivatives is of major interest among synthetic organic chemists. The Huisgen-type copper catalyzed azide-alkyne 1,3-cycloaddition $(\mathrm{Cu}-$ AAC) reaction is the most efficient method for the synthesis of 1,2,3-triazole derivatives, which have found widespread industrial applications as agro chemicals, dyes, corrosion inhibitors, optical brighteners, photographic materials, etc. [4-6]. This method for the synthesis of 1,2,3-triazole derivatives mimics the approach used by nature to generate important compounds. Since its discovery in 2002, a number of "CuAAC" procedures have been developed for making the synthesis of 1,2,3triazoles more efficient and environmentally friendly, and mechanisms of 'click' reactions have matured [7]. In most of the "CuAAC" reactions, the $\mathrm{Cu}$ (1) species is generated from $\mathrm{CuSO}_{4}$ and sodium ascorbate; however, the main problem with this method is the requirement of a large quantity of the metal catalyst, which makes its removal difficult at the later stage [8]. Recently, significant attention also has been paid to the use of copper nanoparticles (CuNPs) as catalysts in "CuAAC" reactions in the synthesis of 1,2,3-triazoles; however, most of these reactions require reductants, organic solvents and high loading of CuNPs [9-11].

Taking into account the great potential of triazoles as valuable building blocks for drug design, the substitution of hydrogen for fluorine in organic compounds is widely used to modify the physical, chemical and biological characteristics of such compounds [12-13]; however, fluorinecontaining triazoles have received much less attention so far [14]. Therefore, in the present work, we wish to report a straightforward, practical procedure for the three-component reaction of $O$-tosyl carbohydrate derivatives, sodium azide and terminal alkynes, catalyzed by copper nanoparticles.

\section{EXPERIMENTAL PROCEDURE}

\subsection{General}

Flash chromatography was performed using silica gel (Merck, Kenilorth, NJ, USA; pore size 60 $\AA$, particle size $0.040-0.063 \mathrm{~mm}$ ). All anhydrous reactions were performed under nitrogen using anhydrous solvents. NMR spectra were obtained on a Bruker (Woodlands, TX, USA) AC $300 \mathrm{spec}-$ trometer operating at $300 \mathrm{MHz}$ for ${ }^{1} \mathrm{H}$ and at 75.47 $\mathrm{MHz}$ for ${ }^{13} \mathrm{C}$. Melting points were determined on a Buchi-510 (Kitmondo, USA) capillary melting point apparatus. Chemical shifts are given in parts per million relative to tetramethylsilane (TMS), and the coupling constants ( $J$ values) are given in hertz $(\mathrm{Hz})$. The spectra were recorded using $\mathrm{CDCl}_{3}$ as the solvent at room temperature. Elemental analysis was recorded on a Perkin-Elmer 240B microanalyzer. Mass spectra were recorded on a Finnigan LCQ DECA XP plus. TEM images were recorded with a JEOL JEM 3010 instrument operated at an accelerating voltage of $300 \mathrm{kV}$.

\subsection{Preparation of copper nanoparticles (CuNPs)}

CuNPs were prepared according to previously published procedures [10] with some modifications. A $0.01 \mathrm{M}$ solution of $\mathrm{CuSO}_{4} \cdot 5 \mathrm{H}_{2} \mathrm{O}$ in ethylene glycol $(3 \mathrm{ml})$ was mixed with $3 \mathrm{ml}$ of an ethylene glycol solution containing $0.005 \mathrm{M} \mathrm{NaOH}$ and $0.015 \mathrm{M} \mathrm{N}_{2} \mathrm{H}_{4} \cdot \mathrm{H}_{2} \mathrm{O}$ under magnetic stirring. The mixture was reacted under microwave irradiation at $100 \mathrm{~W}$ and ultrasound at $50 \mathrm{~W}$ for five minutes, and turned from blue to brick red. The mixture was cooled to room temperature, distilled water was added, and the solution was filtered. The resulting solid was repeatedly washed with distilled water, ethanol and acetone. The resulting solid was placed in a vacuum oven, dried at $60{ }^{\circ} \mathrm{C}$ for $2 \mathrm{hrs}$ and used directly.

\subsection{General procedure for the synthesis of 1,2,3-triazoles}

Sodium azide (0.6 mmol), tosyl-carbohydrates $(0.6 \mathrm{mmol})$ and 4-phenyl-3-prop-2-ynyloxazolidin-2-one $(0.5 \mathrm{mmol})$ were added into a suspension of $0.025 \mathrm{mmol}$ of CuNPs in methanol $(2 \mathrm{ml})$. The reaction mixture was stirred at room temperature until completion, which was monitored by TLC. Then the solution was filtered by suction and the solvent was evaporated under reduced pressure. The residue was subjected to flash column chromatography on silica gel to attain the pure product.

$\left(2^{\prime} R, 4^{\prime} R, 5^{\prime} S, 5^{\prime} R\right)$ Acetic acid 4,5-diacetoxy-6-[4(2-oxo-5-phenyl-oxazolidin-3-ylmethyl)-[1,2,3]triazol-1-ylmethyl]-tetrahydro-pyran-2-yl ester 4a Yield $(90 \%)$, white solid. $T_{\mathrm{m}}=179-180^{\circ} \mathrm{C},[\alpha]^{22} \mathrm{D}$ $=55\left(\mathrm{c} 1, \mathrm{CH}_{2} \mathrm{Cl}_{2}\right), \mathrm{Rf}=0.35$ (cyclohexane/EtOAc 3:7). IR ( $\mathrm{KBr}$ ) umax $/ \mathrm{cm}^{-1}$ : 1645 (triazole $\mathrm{C}=\mathrm{C}$ ); 1480 (triazole $\mathrm{N}=\mathrm{N}) ; 1730(\mathrm{C}=\mathrm{O}) . \mathbf{N M R}^{1} \mathbf{H}(\mathbf{4 0 0}$ MHz, $\left.\mathbf{C D C l}_{3}\right): \delta(\mathrm{ppm}) 2.09\left(\mathrm{~s}, 3 \mathrm{H}, \mathrm{CH}_{3}\right) ; 2.11(\mathrm{~s}$, $\left.3 \mathrm{H}, \mathrm{CH}_{3}\right) ; 2.19$ (s, 3H, $\left.\mathrm{CH}_{3}\right) ; 2.86\left(\mathrm{~m}, 2 \mathrm{H}, \mathrm{H}-3^{\prime}\right)$; $3.31\left(\mathrm{~s}, 2 \mathrm{H}, \mathrm{NCH}_{2}\right) ; 3.41\left(\mathrm{dd}, 1 \mathrm{H}, J 6^{\prime}-6^{\prime \prime} \mathrm{a}=6.5\right.$, $\left.J 6^{\prime \prime} \mathrm{b}-6^{\prime \prime} \mathrm{a}=13.0 \mathrm{~Hz}, \mathrm{H}-6^{\prime \prime} \mathrm{a}\right) ; 3.42$ (dd, 1H, J6'-6"b $\left.=3.0 \mathrm{~Hz}, \mathrm{H}-6^{\prime \prime} \mathrm{b}\right) ; 3.77$ (ddd, $1 \mathrm{H}, J 5^{\prime}-6^{\prime}=10.0 \mathrm{~Hz}$, 
$\left.\mathrm{H}-6^{\prime}\right) ; 4.12\left(\mathrm{dd}, 1 \mathrm{H}, J 5 \mathrm{a}^{\prime \prime}-5 \mathrm{~b}^{\prime \prime}=7.8, J 5^{\prime \prime}-4^{\prime \prime}=8.7, \mathrm{H}-\right.$ $\left.5 b^{\prime \prime}\right), 4.47$ (m, 1H, H-2'); 4.66 (t, 1H, J5"-4" = 8.7, H$4 ") ; 4.96\left(\mathrm{dd}, 1 \mathrm{H}, J 5 \mathrm{a}^{\prime \prime}-5 \mathrm{~b}^{\prime \prime}=7.8, J 5^{\prime \prime}-4 "=8.7, \mathrm{H}-\right.$ $\left.5 \mathrm{a}^{\prime \prime}\right) ; 5.11\left(\mathrm{dd}, 1 \mathrm{H}, J 4^{\prime}-5^{\prime}=10.0 \mathrm{~Hz}, \mathrm{H}-5^{\prime}\right) ; 5.41(\mathrm{~m}$, $\left.1 \mathrm{H}, \mathrm{H}-4^{\prime}\right) ; 7.31-7.42$ (m, 5H, Har); 7.70 (s, 1H, H-5). NMR ${ }^{13} \mathbf{C}\left(\mathbf{1 0 0 . 6} \mathbf{M H z}, \mathbf{C D C l}_{3}\right): \delta(\mathrm{ppm}) 21.3$ $\left(\mathrm{CH}_{3}\right) ; 21.4\left(\mathrm{CH}_{3}\right) ; 22.1\left(\mathrm{CH}_{3}\right) ; 23.5\left(\mathrm{C}-2^{\prime}\right) ; 47.9$ $(\mathrm{CN}) ; 69.1\left(\mathrm{C}-5^{\prime}\right) ; 70.3\left(\mathrm{C}-5^{\prime \prime}\right) ; 73.3\left(\mathrm{C}-4^{\prime \prime}\right) ; 73.9(\mathrm{C}-$ 4'); 74.7 (C-6'); 88.3 (C-3'); 124.1 (C-4); 126.8 (Car); 129.1 (C-ar); 129.9 (C-ar ); 136.1 (C-ar); 143.7 (C-5); 166.3 (CO); 170.1 (CO); 171.0 (CO); 171.4 (CO). HRMS Calcd for $\mathrm{C}_{24} \mathrm{H}_{28} \mathrm{~N}_{4} \mathrm{O}_{9} 516.1856$. Found: 516.1848. Elemental analysis: $\mathrm{C}_{24} \mathrm{H}_{28} \mathrm{~N}_{4} \mathrm{O}_{9}$ requires $\mathrm{C}, 55.81 ; \mathrm{H}, 5.46 ; \mathrm{N}, 10.85 \%$; found $\mathrm{C}, \mathrm{C}$, $55.78 ; \mathrm{H}, 5.44 ; \mathrm{N}, 10.83 \%$.

(4'R, 2'R, 3'S, 4'S, 5'R) Acetic acid 2',5'diacetoxy-3'-fluoro-6-[4"-(2"-oxo-4-phenyl-oxazolidin-3"-ylmethyl)-[1,2,3]triazol-1-ylmethyl]-tetrahydro-pyran-4'-yl ester $4 \mathrm{~b}$

Yield $(75 \%)$, white solid. $T_{\mathrm{m}}=162-163{ }^{\circ} \mathrm{C},[\alpha]_{\mathrm{D}}^{22}$ $=37\left(\mathrm{c} 1, \mathrm{CH}_{2} \mathrm{Cl}_{2}\right), \mathrm{Rf}=0.4$ (cyclohexane/EtOAc 3:7). IR ( $\mathrm{KBr}$ ) umax $/ \mathrm{cm}^{-1}$ : 1640 (triazole $\mathrm{C}=\mathrm{C}$ ); 1475 (triazole $\mathrm{N}=\mathrm{N}) ; 1730(\mathrm{C}=\mathrm{O}) . \mathbf{N M R}{ }^{1} \mathbf{H}(\mathbf{4 0 0}$ MHz, $\left.\mathbf{C D C l}_{3}\right): \delta(\mathrm{ppm}) 2.12\left(\mathrm{~s}, 3 \mathrm{H}, \mathrm{CH}_{3}\right) ; 2.14(\mathrm{~s}$, $\left.3 \mathrm{H}, \mathrm{CH}_{3}\right) ; 2.20\left(\mathrm{~s}, 3 \mathrm{H}, \mathrm{CH}_{3}\right) ; 3.33\left(\mathrm{~s}, 2 \mathrm{H}, \mathrm{NCH}_{2}\right)$; 3.43 (dd, 1H, J6'-6"a = 6.5, J6" b-6"a = 13.0 Hz, H6"a); 3.45 (dd, 1H, J6'-6"b = 3.0 Hz, H-6"b); 3.79 (ddd, 1H, J5'-6' = $\left.10.0 \mathrm{~Hz}, \mathrm{H}-6^{\prime}\right) ; 4.14(\mathrm{dd}, 1 \mathrm{H}$, $\left.J 5 \mathrm{a}^{\prime \prime}-5 \mathrm{~b}^{\prime \prime}=7.8, J 5^{\prime \prime}-4^{\prime \prime}=8.7, \mathrm{H}-5 \mathrm{~b}^{\prime \prime}\right), 4.47$ (ddd, 1H, $\left.J 2^{\prime}-3^{\prime}=4.0, J 3^{\prime}-4^{\prime}=10.0, J 3^{\prime}-\mathrm{F}=53.0 \mathrm{~Hz}, \mathrm{H}-3^{\prime}\right)$; 4.66 (t, 1H, J5"-4" = 8.7, H-4"); 4.96 (dd, 1H, J5a"$\left.5 b^{\prime \prime}=7.8, J 5^{\prime \prime}-4^{\prime \prime}=8.7, \mathrm{H}-5 \mathrm{a}^{\prime \prime}\right) ; 5.14\left(\mathrm{dd}, 1 \mathrm{H}, J 4^{\prime}-5^{\prime}\right.$ $\left.=10.0 \mathrm{~Hz}, \mathrm{H}-5^{\prime}\right) ; 5.44\left(\mathrm{ddd}, 1 \mathrm{H}, J 4^{\prime}-\mathrm{F}=14.0 \mathrm{~Hz}, \mathrm{H}-\right.$ 4'); $5.86\left(\mathrm{dd}, 1 \mathrm{H}, J 2^{\prime}-\mathrm{F}=3.0 \mathrm{~Hz}, \mathrm{H}-2^{\prime}\right) ; 7.33-7.45$ (m, 5H, Har); 7.75 (s, 1H, H-5). NMR ${ }^{13} \mathbf{C}(\mathbf{1 0 0 . 6}$ MHz, $\left.\mathbf{C D C l}_{3}\right): \delta(\mathrm{ppm}) 21.2\left(\mathrm{CH}_{3}\right) ; 21.6\left(\mathrm{CH}_{3}\right)$; $22.3\left(\mathrm{CH}_{3}\right) ; 47.7(\mathrm{CN}) ; 69.2\left(\mathrm{~d}, J 5^{\prime}-\mathrm{F}=7 \mathrm{~Hz}, \mathrm{C}-5^{\prime}\right)$; 70.1 (C-5"); 73.3 (C-4"); 73.7 (d, J4'-F = $20 \mathrm{~Hz}, \mathrm{C}-$ 4'); 74.8 (C-6'); 88.7 (d, J3'-F = $\left.196 \mathrm{~Hz}, \mathrm{C}-3^{\prime}\right) ; 91.5$ (d, $\left.J 2^{\prime}-\mathrm{F}=23 \mathrm{~Hz}, \mathrm{C}-2^{\prime}\right) ; 124.3$ (C-4); 127.1 (C-ar); 129.2 (C-ar); 129.6 (C-ar); 136.7 (C-ar); 144.2 (C-5); 166.1 (CO); 170.5 (CO); 171.2 (CO); 171.4 (CO). NMR ${ }^{19}$ F (235 MHz, $\left.\mathbf{C D C l}_{3}\right): \delta(\mathrm{ppm})$ - 206.4. HRMS Calcd for $\mathrm{C}_{24} \mathrm{H}_{27} \mathrm{FN}_{4} \mathrm{O}_{9}$ 534.1762. Found: 534.1759. Elemental analysis: $\mathrm{C}_{24} \mathrm{H}_{27} \mathrm{FN}_{4} \mathrm{O}_{9}$ requires $\mathrm{C}, 53.93 ; \mathrm{H}, 5.09 ; \mathrm{N}, 10.48 \%$; found $\mathrm{C}$, $53.90 ; \mathrm{H}, 5.05 ; \mathrm{N}, 10.43 \%$.

$\left(4^{\prime \prime} R, 2^{\prime} S, 3^{\prime} S, 4^{\prime} S, 5^{\prime} R\right)$ Acetic acid 2',5'-diacetoxy3'-fluoro-6-[4"-(2"-oxo-4-phenyl-oxazolidin-3"ylmethyl)-[1,2,3]triazol-1-ylmethyl]-tetrahydropyran-4'-yl ester 4c

Yield $(70 \%)$, white solid. $T_{\mathrm{m}}=156-166^{\circ} \mathrm{C},[\alpha]^{22}{ }_{\mathrm{D}}$ $=41\left(\mathrm{c} 1, \mathrm{CH}_{2} \mathrm{Cl}_{2}\right), \mathrm{Rf}=0.3($ cyclohexane $/$ EtOAc 3:7). IR ( $\mathrm{KBr}$ ) $u \max / \mathrm{cm}^{-1}$ : 1645 (triazole $\mathrm{C}=\mathrm{C}$ );
1475 (triazole $\mathrm{N}=\mathrm{N}) ; 1735(\mathrm{C}=\mathrm{O}) . \quad \mathbf{N M R}^{\mathbf{1}} \mathbf{H}(\mathbf{4 0 0}$ MHz, $\left.\mathbf{C D C l}_{3}\right): \delta$ (ppm) 2.09 (s, 3H, $\left.\mathrm{CH}_{3}\right) ; 2.12$ (s, $\left.3 \mathrm{H}, \mathrm{CH}_{3}\right) ; 2.21\left(\mathrm{~s}, 3 \mathrm{H}, \mathrm{CH}_{3}\right) ; 3.32\left(\mathrm{~s}, 2 \mathrm{H}, \mathrm{NCH}_{2}\right)$; 3.44 (dd, $1 \mathrm{H}, J 6^{\prime}-6^{\prime \prime} \mathrm{a}=6.5, J 6^{\prime \prime} \mathrm{b}-6^{\prime \prime} \mathrm{a}=13.0 \mathrm{~Hz}, \mathrm{H}-$ 6"a); 3.45 (dd, 1H, J6'-6"b = 3.0 Hz, H-6"b); 3.80 (ddd, 1H, J5'-6' = $\left.10.0 \mathrm{~Hz}, \mathrm{H}-6^{\prime}\right) ; 4.13(\mathrm{dd}, 1 \mathrm{H}$, $\left.J 5 \mathrm{a}^{\prime \prime}-5 \mathrm{~b}^{\prime \prime}=7.8, J 5^{\prime \prime}-4^{\prime \prime}=8.7, \mathrm{H}-5 \mathrm{~b}^{\prime \prime}\right), 4.47$ (ddd, $1 \mathrm{H}, J 2^{\prime}-3^{\prime}=8.0, J 3^{\prime}-4^{\prime}=10.0, J 3^{\prime}-\mathrm{F}=53.0 \mathrm{~Hz}, \mathrm{H}-$ 3'); 4.67 (t, 1H, J5"-4" = 8.7, H-4"); $4.93(\mathrm{dd}, 1 \mathrm{H}$, $\left.J 5 \mathrm{a}^{\prime \prime}-5 \mathrm{~b}^{\prime \prime}=7.8, J 5^{\prime \prime}-4^{\prime \prime}=8.7, \mathrm{H}-5 \mathrm{a} "\right) ; 5.15(\mathrm{dd}, 1 \mathrm{H}$, $\left.J 4^{\prime}-5^{\prime}=10.0 \mathrm{~Hz}, \mathrm{H}-5^{\prime}\right) ; 5.40\left(\mathrm{ddd}, 1 \mathrm{H}, J 4^{\prime}-\mathrm{F}=14.0\right.$ $\left.\mathrm{Hz}, \mathrm{H}-4^{\prime}\right) ; 5.81\left(\mathrm{dd}, 1 \mathrm{H}, J 2^{\prime}-\mathrm{F}=3.0 \mathrm{~Hz}, \mathrm{H}-2^{\prime}\right)$; 7.32-7.47 (m, 5H, Har); 7.71 (s, 1H, H-5). NMR ${ }^{13} \mathbf{C}$ (100.6 $\left.\mathbf{M H z}, \mathbf{C D C l}_{3}\right): \delta(\mathrm{ppm}) 21.1\left(\mathrm{CH}_{3}\right)$; $21.3\left(\mathrm{CH}_{3}\right) ; 22.1\left(\mathrm{CH}_{3}\right) ; 48.0(\mathrm{CN}) ; 68.9\left(\mathrm{~d}, J 5^{\prime}-\mathrm{F}=\right.$ $\left.7 \mathrm{~Hz}, \mathrm{C}-5^{\prime}\right) ; 70.1\left(\mathrm{C}-5^{\prime \prime}\right) ; 73.2\left(\mathrm{C}-4^{\prime \prime}\right) ; 73.5$ (d, J4'-F $\left.=20 \mathrm{~Hz}, \mathrm{C}-4^{\prime}\right) ; 74.5\left(\mathrm{C}-6^{\prime}\right) ; 88.6\left(\mathrm{~d}, J 3^{\prime}-\mathrm{F}=196\right.$ Hz, C-3'); 91.1 (d, J2'-F = $\left.23 \mathrm{~Hz}, \mathrm{C}-2^{\prime}\right) ; 123.7$ (C4); 126.5 (C-ar); 129.3 (C-ar); 129.6 (C-ar ); 136.5 (C-ar); 144.0 (C-5); 165.9 (CO); 171.0 (CO); 171.2 (CO); 171.3 (CO). NMR ${ }^{19}$ F (235 $\left.\mathbf{~ M H z}, \mathbf{C D C l}_{3}\right)$ : $\delta$ (ppm) - 206.6. HRMS Calcd for $\mathrm{C}_{24} \mathrm{H}_{27} \mathrm{FN}_{4} \mathrm{O}_{9}$ 534.1762. Found: 534.1757. Elemental analysis: $\mathrm{C}_{24} \mathrm{H}_{27} \mathrm{FN}_{4} \mathrm{O}_{9}$ requires $\mathrm{C}, 53.93 ; \mathrm{H}, 5.09 ; \mathrm{N}$, $10.48 \%$; found $\mathrm{C}, 53.95 ; \mathrm{H}, 5.11 ; \mathrm{N}, 10.42 \%$.

$\left(4^{\prime \prime} R, 2^{\prime} R, 3^{\prime} S, 4^{\prime} S, 5^{\prime} R\right)$ Acetic acid 4,5-diacetoxy-3-fluoro-6-\{2-[4-(2-oxo-4-phenyl-oxazolidin3-ylmethyl)-[1,2,3]triazol-1-yl]-ethoxymethyl\}tetrahydro-pyran-2-yl ester 4d

Yield $(85 \%)$, white solid. $T_{\mathrm{m}}=174-175^{\circ} \mathrm{C},[\alpha]^{22}{ }_{\mathrm{D}}$ $=57\left(\mathrm{c} 1, \mathrm{CH}_{2} \mathrm{Cl}_{2}\right), \mathrm{Rf}=0.35$ (cyclohexane/EtOAc 3:7). IR ( $\mathrm{KBr}$ ) umax $/ \mathrm{cm}^{-1}$ : 1645 (triazole $\mathrm{C}=\mathrm{C}$ ); 1470 (triazole $\mathrm{N}=\mathrm{N}) ; 1730(\mathrm{C}=\mathrm{O}) . \mathbf{N M R}^{\mathbf{1}} \mathbf{H}(\mathbf{4 0 0}$ MHz, $\left.\mathbf{C D C l}_{3}\right): \delta(\mathrm{ppm}) 2.10\left(\mathrm{~s}, 3 \mathrm{H}, \mathrm{CH}_{3}\right) ; 2.15$ (s, $\left.3 \mathrm{H}, \mathrm{CH}_{3}\right) ; 2.21\left(\mathrm{~s}, 3 \mathrm{H}, \mathrm{CH}_{3}\right) ; 3.34\left(\mathrm{~s}, 2 \mathrm{H}, \mathrm{NCH}_{2}\right)$; $3.40(\mathrm{dd}, 2 \mathrm{H}, J 7-8=5.0 \mathrm{~Hz}, \mathrm{H}-8) ; 3.44(\mathrm{dd}, 1 \mathrm{H}$, $\left.J 6^{\prime}-6^{\prime \prime} \mathrm{a}=6.5, J 6^{\prime \prime} \mathrm{b}-6^{\prime \prime} \mathrm{a}=13.0 \mathrm{~Hz}, \mathrm{H}-6^{\prime \prime} \mathrm{a}\right) ; 3.44$ (dd, 1H, J6'-6"b = 3.0 Hz, H-6"b); 3.59 (dd, 2H, $J 7-8=5.0 \mathrm{~Hz}, \mathrm{H}-7$ ); 3.79 (ddd, $1 \mathrm{H}, J 5^{\prime}-6^{\prime}=10.0$ $\left.\mathrm{Hz}, \mathrm{H}-6^{\prime}\right)$; 4.15 (dd, $1 \mathrm{H}, J 5 \mathrm{a}^{\prime \prime}-5 \mathrm{~b}^{\prime \prime}=7.8, J 5^{\prime \prime}-4^{\prime \prime}=$ 8.7, H-5b"), 4.45 (ddd, $1 \mathrm{H}, J 2^{\prime}-3^{\prime}=4.0, J 3^{\prime}-4^{\prime}=$ $\left.10.0, J 3^{\prime}-\mathrm{F}=53.0 \mathrm{~Hz}, \mathrm{H}-3^{\prime}\right)$; $4.65\left(\mathrm{t}, 1 \mathrm{H}, J 5^{\prime \prime}-4^{\prime \prime}=\right.$ 8.7, H-4"); 4.94 (dd, $1 \mathrm{H}, J 5 \mathrm{a}^{\prime \prime}-5 \mathrm{~b}^{\prime \prime}=7.8, J 5^{\prime \prime}-4^{\prime \prime}=$ 8.7, H-5a"'); 5.16 (dd, $\left.1 \mathrm{H}, J 4^{\prime}-5^{\prime}=10.0 \mathrm{~Hz}, \mathrm{H}-5^{\prime}\right)$; $5.45\left(\mathrm{ddd}, 1 \mathrm{H}, J 4^{\prime}-\mathrm{F}=14.0 \mathrm{~Hz}, \mathrm{H}-4^{\prime}\right) ; 5.84(\mathrm{dd}$, $\left.1 \mathrm{H}, J 2^{\prime}-\mathrm{F}=3.0 \mathrm{~Hz}, \mathrm{H}-2^{\prime}\right)$; 7.31-7.44 (m, 5H, Har); 7.72 (s, 1H, H-5). NMR ${ }^{13} \mathbf{C}\left(\mathbf{1 0 0 . 6} \mathbf{~ M H z}, \mathbf{C D C l}_{3}\right)$ : $\delta(\mathrm{ppm}) 21.4\left(\mathrm{CH}_{3}\right) ; 21.6\left(\mathrm{CH}_{3}\right) ; 22.3\left(\mathrm{CH}_{3}\right) ; 47.7$ $(\mathrm{CN}) ; 50.8(\mathrm{C}-8) ; 69.4$ (d, J5'-F = $\left.7 \mathrm{~Hz}, \mathrm{C}-5^{\prime}\right) ; 70.2$ $\left(\mathrm{C}-5^{\prime \prime}\right) ; 70.6(\mathrm{C}-7) ; 73.3\left(\mathrm{C}-4^{\prime \prime}\right) ; 73.8\left(\mathrm{~d}, J 4^{\prime}-\mathrm{F}=20\right.$ $\left.\mathrm{Hz}, \mathrm{C}-4^{\prime}\right) ; 74.7$ (C-6'); 88.7 (d, J3'-F = $196 \mathrm{~Hz}, \mathrm{C}-$ 3'); 91.9 (d, $\left.J 2^{\prime}-\mathrm{F}=23 \mathrm{~Hz}, \mathrm{C}-2^{\prime}\right)$; 124.1 (C-4); 126.4 (C-ar); 128.9 (C-ar); 129.5 (C-ar ); 136.5 (Car); 144.1 (C-5); 165.1 (CO); 170.7 (CO); 171.3 
(CO); 171.7 (CO). NMR ${ }^{19} \mathbf{F}\left(235\right.$ MHz, $\left.\mathbf{C D C l}_{3}\right): \delta$ (ppm) - 206.7. HRMS Calcd for $\mathrm{C}_{26} \mathrm{H}_{31} \mathrm{FN}_{4} \mathrm{O}_{10}$ 578.2024. Found: 578.2021. Elemental analysis: $\mathrm{C}_{26} \mathrm{H}_{31} \mathrm{FN}_{4} \mathrm{O}_{10}$ requires $\mathrm{C}, 53.98 ; \mathrm{H}, 5.40 ; \mathrm{N}, 9.68 \%$; found $\mathrm{C}, 53.94 ; \mathrm{H}, 5.36 ; \mathrm{N}, 9.66 \%$

$\left(4^{\prime \prime} R, 2^{\prime} S, 3^{\prime} S, 4^{\prime} S, 5^{\prime} R\right)$ Acetic acid 4,5-diacetoxy-3-fluoro-6-\{2-[4-(2-0x0-4-phenyl-oxazolidin-3-ylmethyl)-[1,2,3]triazol-1-yl]-ethoxymethyl\}-tetrahydro-pyran-2-yl ester 4d

Yield $(90 \%)$, white solid. $T_{\mathrm{m}}=191-192{ }^{\circ} \mathrm{C},[\alpha]^{22}{ }_{\mathrm{D}}$ $=44\left(\mathrm{c} 1, \mathrm{CH}_{2} \mathrm{Cl}_{2}\right), \mathrm{Rf}=0.4$ (cyclohexane/EtOAc 3:7). IR ( $\mathrm{KBr})$ vmax $/ \mathrm{cm}^{-1}$ : 1640 (triazole $\left.\mathrm{C}=\mathrm{C}\right)$; 1470 (triazole $\mathrm{N}=\mathrm{N}) ; 1730(\mathrm{C}=\mathrm{O}) . \mathbf{N M R}{ }^{\mathbf{1}} \mathbf{H}(\mathbf{4 0 0}$ $\left.\mathbf{M H z}, \mathbf{C D C l}_{3}\right): \delta(\mathrm{ppm}) 2.08\left(\mathrm{~s}, 3 \mathrm{H}, \mathrm{CH}_{3}\right) ; 2.10(\mathrm{~s}$, $\left.3 \mathrm{H}, \mathrm{CH}_{3}\right) ; 2.20\left(\mathrm{~s}, 3 \mathrm{H}, \mathrm{CH}_{3}\right) ; 3.31\left(\mathrm{~s}, 2 \mathrm{H}, \mathrm{NCH}_{2}\right)$; 3.43 (dd, 1H, J6'-6"a = 6.5, J6" b-6"a = 13.0 Hz, H6"a); 3.44 (dd, 1H, J6'-6"b = 3.0 Hz, H-6"b); 3.79 (ddd, 1H, J5'-6' = $\left.10.0 \mathrm{~Hz}, \mathrm{H}-6^{\prime}\right) ; 4.12(\mathrm{dd}, 1 \mathrm{H}$, $\left.J 5 \mathrm{a}^{\prime \prime}-5 \mathrm{~b}^{\prime \prime}=7.8, J 5^{\prime \prime}-4^{\prime \prime}=8.7, \mathrm{H}-5 \mathrm{~b}^{\prime \prime}\right), 4.45$ (ddd, $1 \mathrm{H}, J 2^{\prime}-3^{\prime}=8.0, J 3^{\prime}-4^{\prime}=10.0, J 3^{\prime}-\mathrm{F}=53.0 \mathrm{~Hz}, \mathrm{H}-$ 3'); 4.66 (t, 1H, J5"-4" = 8.7, H-4"); $4.92(\mathrm{dd}, 1 \mathrm{H}$, $\left.J 5 \mathrm{a}^{\prime \prime}-5 \mathrm{~b}^{\prime \prime}=7.8, J 5^{\prime \prime}-4^{\prime \prime}=8.7, \mathrm{H}-5 \mathrm{a}^{\prime \prime}\right) ; 5.14(\mathrm{dd}, 1 \mathrm{H}$, $\left.J 4^{\prime}-5^{\prime}=10.0 \mathrm{~Hz}, \mathrm{H}-5^{\prime}\right) ; 5.39$ (ddd, $1 \mathrm{H}, J 4^{\prime}-\mathrm{F}=14.0$ $\left.\mathrm{Hz}, \mathrm{H}-4^{\prime}\right) ; 5.79$ (dd, $\left.1 \mathrm{H}, J 2^{\prime}-\mathrm{F}=3.0 \mathrm{~Hz}, \mathrm{H}-2^{\prime}\right)$; 7.35-7.49 (m, 5H, Har); 7.75 (s, 1H, H-5). NMR ${ }^{13} \mathbf{C}\left(\mathbf{1 0 0 . 6} \mathbf{M H z}, \mathbf{C D C l}_{3}\right): \delta(\mathrm{ppm}) 21.3\left(\mathrm{CH}_{3}\right)$; $21.3\left(\mathrm{CH}_{3}\right) ; 22.2\left(\mathrm{CH}_{3}\right) ; 48.0(\mathrm{CN}) ; 68.7\left(\mathrm{~d}, J 5^{\prime}-\mathrm{F}=\right.$ $\left.7 \mathrm{~Hz}, \mathrm{C}-5^{\prime}\right) ; 70.2$ (C-5"); 73.3 (C-4"); 73.5 (d, J4'-F $\left.=20 \mathrm{~Hz}, \mathrm{C}-4^{\prime}\right) ; 74.6\left(\mathrm{C}-6^{\prime}\right) ; 88.3\left(\mathrm{~d}, J 3^{\prime}-\mathrm{F}=196\right.$ $\left.\mathrm{Hz}, \mathrm{C}-3^{\prime}\right) ; 91.3$ (d, J2'-F = $\left.23 \mathrm{~Hz}, \mathrm{C}-2^{\prime}\right) ; 123.2$ (C4); 127.1 (C-ar); 128.9 (C-ar); 129.3 (C-ar ); 136.3 (C-ar); 144.1 (C-5); 165.7 (CO); 171.1 (CO); 171.6 (CO); 171.4 (CO). NMR ${ }^{19} \mathbf{F}$ (235 $\left.\mathbf{~ M H z}, \mathbf{C D C l}_{3}\right)$ : $\delta(\mathrm{ppm})$ - 206.7. HRMS Calcd for $\mathrm{C}_{26} \mathrm{H}_{31} \mathrm{FN}_{4} \mathrm{O}_{10}$ 578.2020. Found: 578.2024. Elemental analysis: $\mathrm{C}_{26} \mathrm{H}_{31} \mathrm{FN}_{4} \mathrm{O}_{10}$ requires $\mathrm{C}, 53.98 ; \mathrm{H}, 5.40 ; \mathrm{N}$, 9.68\%; found C, 53.90; H, 5.35; N, 9.64\%

\section{RESULTS AND DISCUSSION}

Copper nanoparticles were prepared by reducing copper sulfate pentahydrate with hydrazine hydrate in ethylene glycol under combined of microwave irradiation $(100 \mathrm{~W})$ and ultrasound (50 W) at $120-125{ }^{\circ} \mathrm{C}$ for $5 \mathrm{~min}$. This method provided stable CuNPs with a narrow particle size distribution that can be kept for several months in air. The copper nanoparticles were characterized by Xray diffraction (XRD) and transmission electron microscopy (TEM) [15]. The powder X-ray diffraction (XRD) studies of the precipitates showed the presence of diffraction peaks located at $2 \theta$ values of 43.6, 50.4 and 73.8 (Fig. 1A), which suggested the formation of pure copper. Thus, XRD studies confirm the formation of CuNPs. The TEM image of CuNPs showed the presence of nanospheres. The resulting particle size was found to be 18 to $23 \mathrm{~nm}$ (Fig. 1B).

For our initial study, we chose $(R)$-4-phenyl-3prop-2-ynyl-oxazolidin-2-one 1 , acetic acid 4,6-diacetoxy-2-(toluene-4-sulfonyloxymethyl)-tetrahydropyran-3-yl ester $\mathbf{2 a}$ and sodium azide $\mathbf{3}$ to optimize the reaction conditions. The results are shown in Table 1. We found that all of the $\mathrm{Cu}(\mathrm{I})$ and $\mathrm{Cu}(\mathrm{II})$ salts could effectively catalyze the click reaction of $(R)$-4-phenyl-3-prop-2-ynyl-oxazolidin-2-one 1 and acetic acid 4,6-diacetoxy-2-(toluene-4-sulfonyloxymethyl)-tetrahydro-pyran-3-yl ester 2a to obtain 1,2,3-triazole 4a exclusively in good yields in methanol at room temperature (Table 1, entries 14). When CuNPs were used, an excellent yield $(90 \%)$ was achieved with the same amount of the catalysts (5 mol \%, Table 1, entry 5).
A

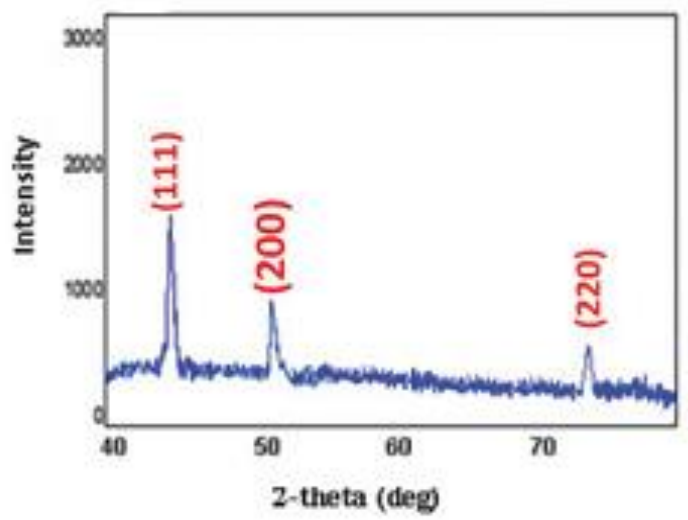

B

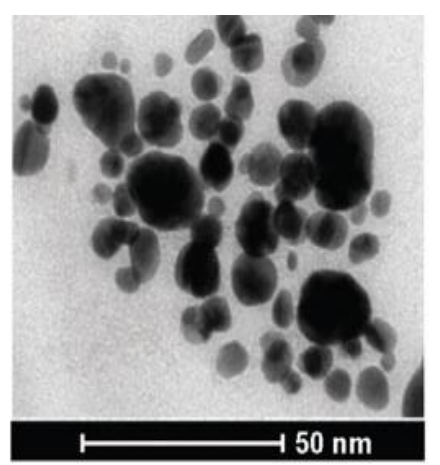

Fig. 1. XRD pattern (A) and TEM images (B) of CuNPs 
Table 1

Screening of the catalyst for the three-component click reaction<smiles>C#CCN1C(=O)OC[C@H]1Pc1ccccc1</smiles>

1

2a

\begin{tabular}{ccc}
\hline \hline Entry & Catalyst & Yield (\%) \\
\hline $\mathbf{1}$ & $\mathrm{CuBr}$ & 40 \\
$\mathbf{2}$ & $\mathrm{CuCl}$ & 50 \\
$\mathbf{3}$ & $\mathrm{CuSO}_{4} 5 \mathrm{H}_{2} \mathrm{O}$ & 70 \\
$\mathbf{4}$ & $\mathrm{CuO}$ & 65 \\
$\mathbf{5}^{\mathbf{a}}$ & $\mathrm{CuNPs}$ & 90 \\
$\mathbf{6}^{\mathbf{b}}$ & $\mathrm{CuNPs}$ & 85 \\
$\mathbf{7}^{\mathbf{c}}$ & $\mathrm{CuNPs}$ & 80 \\
\hline \hline
\end{tabular}

${ }^{\text {a }}$ Catalyst $(0.05 \mathrm{mmol})$.

${ }^{\mathrm{b}}$ Catalyst $(0.03 \mathrm{mmol})$.

${ }^{\mathrm{c}}$ Catalyst (0.01 mmol).

Table 2

Azide-alkyne cycloaddition catalyzed with CuNPs<smiles>C#CCN1C(=O)OC[C@H]1c1ccccc1</smiles>

1

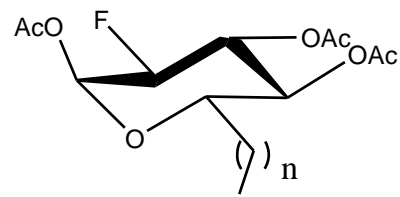

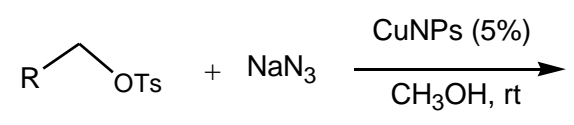

$2 b-e$<smiles>Cn1cc(CN2C(=O)OC[C@@H]2c2ccccc2)nn1</smiles>

4b-e

\begin{tabular}{ccc}
\hline \hline Triazole & $\mathrm{n}$ & Yield (\%) \\
\hline $\mathbf{4 b}$ & 1 & 75 \\
$\mathbf{4 c}$ & 1 & 70 \\
$\mathbf{4 d}$ & 2 & 85 \\
$\mathbf{4 e}$ & 2 & 90 \\
\hline
\end{tabular}


This illustrates that the particle size and surface properties played an essential role in this transformation. Next, we studied the loading of CuNPs and the results showed that $5 \mathrm{~mol} \%$ of CuNPs is the optimized quantity (Table 1 , entries 5, 6 and 7). This showed that steric hindrance of the carbohydrate also has no significant effect on the reaction [16].

Under the optimized conditions, different carbohydrates were used in this click reaction and an array of 1,4-disusbstituted 1,2,3-triazoles were synthesized efficiently in good to excellent yields. These results are presented in Table 2. The length of the alkyl chain did not noticeably affect the yield of the product (Table 2, entries 1, 5, and 9). In all cases the cycloaddition was accomplished with complete regioselectivity.

\section{CONCLUSION}

In conclusion, we have developed a simple and highly efficient one-pot method to synthesize complex 1,2,3-triazoles regioselectively from organic tosyl carbohydrates, terminal alkynes, and sodium azide. The reactions were performed under ambient conditions in methanol at room temperature, using CuNPs as catalysts.

\section{REFERENCE}

[1] M. Meldal, C. W. Tornøe, Cu-catalyzed azidealkyne cycloaddition. Chem. Rev., 108, 2952-3015 (2008).

[2] R. K. Iha, K. L. Wooley, A. M. Nystrom, D. J. Burke, M. J. Kade, C. J. Hawker, Applications of orthogonal "click" chemistries in the synthesis of functional soft materials, Chem. Rev., 109, 56205686 (2009).

[3] P. Thirumurugan, D. Matosiuk, K. Jozwiak, Click chemistry for drug development and diverse chemical-biology applications, Chem. Rev., 113, 49054979 (2012).

[4] J. E. Moses, A. D. Moorhouse, The growing applications of click chemistry, Chem. Soc. Rev., 36, 1249-1262 (2007).

[5] J. F. Lutz, 1,3-Dipolar Cycloadditions of Azides and Alkynes: A Universal Ligation Tool in Polymer and Materials Science, Angew. Chem., Int. Ed., 46, 1018-1025 (2007).
[6] C. W. Tornoe, C. Christensen, M. Meldal, Peptidotriazoles on Solid Phase: [1,2,3]-Triazoles by Regiospecific Copper(I)-Catalyzed 1,3-Dipolar Cycloadditions of Terminal Alkynes to Azides, $J$. Org. Chem., 67, 3057-3064 (2002).

[7] M.-L. Teyssot, A. Chevry, M. Traikia, M. ElGhozzi, D. Avignant, A. Gautier, Improved Copper(I)-NHC Catalytic Efficiency on Huisgen Reaction by Addition of Aromatic Nitrogen Donors, Chem. Eur. J., 15, 6322-6326 (2009).

[8] C. Ornelas, J. Ruiz, E. Cloutet, S. Alves, D. Astruc, Click Assembly of 1,2,3-Triazole-Linked Dendrimers, Including Ferrocenyl Dendrimers, which Sense Both Oxo Anions and Metal Cations, Angew. Chem., Int. Ed., 46, 872-877 (2007).

[9] A. Kumar, S. Aerry, A. Saxena, A. Deb, S. Mozumdar, Copper nanoparticulates in Guar-gum: a recyclable catalytic system for the Huisgen $[3+$ 2]-cycloaddition of azides and alkynes without additives under ambient conditions, Green Chem., 14, 1298-1301 (2012).

[10] R. Hudson, C.-J Li, A. Moores, Magnetic copperiron nanoparticles as simple heterogeneous catalysts for the azide-alkyne click reaction in water, Green Chem., 14, 622-624 (2012).

[11] F. Alonso, Y. Moglie, G. Radivoy, M. Yus, Click chemistry from organic halides, diazonium salts and anilines in water catalysed by copper nanoparticles on activated carbon, Org. Biomol. Chem., 9, 6385-6395 (2011).

[12] X.-L. Qiu, W.-D. Meng, F.-L. Qing, Synthesis of fluorinated amino acids, Tetrahedron 60, 67116745 (2004).

[13] N. Ben Hamadi, Asymmetric synthesis of enantiopure fluorinated cyclopropyl carbohydrate analogues, J. Fluorine Chem. 170, 47-51 (2015).

[14] C. Hager, R. Miethchen, H. Reinke, Organofluorine compounds and fluorinating agents, Part 26: New reversed nucleosides - perfluoroalkyl substituted 1,2,3-triazoles linked to D-galactose and Daltrose, J. Fluorine Chem. 104, 135-142 (2000).

[15] H. Zhu, C. Zhang, Y. Yin, Novel synthesis of copper nanoparticles: influence of the synthesis conditions on the particle size. Nanotechnology 16, 3079-3083 (2005).

[16] K. Hajlaoui, N. Ben Hamadi, M. Moncef, Copper nanoparticles cycloaddition of terminal acetylenes with carbohydrate azide, Catal Lett., 145, 12461250 (2015). 